\title{
LÉGISLATION
}

\section{Le Mois Fiscal}

par Roger et Jacques Lefebvre

\section{NOUS VOUS SIGNALONS QUE:}

Par application de l'article 7 des nouvelles loi; codifiées par le décret de réforme fiscale du 20 juillet 1934, l'impôt sur les bénéfices industriels et commerciaux reposera désormais sur "le bénéfice net déterminé d'après les résultats d'ensemble des opérations de toute nature effectuées par les en reprises y compris les cessions d'éléments quelconques de l'actif, soit en cours, soit en fin d'exploitation".

La disposition incluse dans ce texte, qui prévoit l'imposition des plus-values réalisées "en fin d'exploitation ", c'est-à-dire à l'occasion de la cessation ou de la cession de l'entreprise, sera applicable pour la première fois lors de l'établissement de l'impôt dû au titre de l'année 1935.

Par ailleurs, on sait qu'en fin d'exploitation l'impôt sur les bénéfices industriels et commerciaux dû en raison des bénéfices qui n'ont pas encore été taxés est immédiatement établi, au titre de l'année même de la cessation (et non de l'année suivante) et selon les règles en vigueur de ladite année.

Il résulte de ces dispositions combinées que les impositions qui frapperont les bénéfices réalisés au cours de leur dernier exercice par les entreprises qui cèderont ou cesseront leur exploitation avant le $1^{\text {er }}$ janvier 1935 seront établies au titre de l'année 1934 et que, par suite, les plus-values que cette cession ou cessation pourra faire apparaittre ne seront pas atteintes par l'impôt.

$$
* *
$$

Par application du principe de la " confusion des patrimoines ", lés gérants des sociétés en commandite par actions étaient jusqu'alors assujettis à l'impôt général sur le revenu, d'après la part leur revenant dans les bénéfices réalisés par la société que ces bénéfices aient été distribués ou mis en réserve (arrêts du Conseil d'Etat du 8 avril 1932 et 31 mars 1933).

L'article 113 des nouvelles lois codifiées far le décret du 20 juillet 1934 modifie cet état de choses à partir de 1935 (revenus de 1934).

Désormais le patrimoine des gérants et celui de la société ne seront plus confondus. Les associés-gérants ne se:ont imposés sur leur part dans les bénéfices mis en réserve qu'au moment de la mise en distribution de ces réserves. Par contre, les sommes qui leur sont allouées à titre de rémunération de leurs fonctions ou de leurs apports seront comprises dans les bases de l'impôt général même si l'exercice social est déficitaire.

$$
*^{*} *
$$

La patente n'est pas due par une entreprise commerciale en liguidation qui n'a plus fait aucun acte de commerce depuis le 1er janvier, mais qui s'est bornée, avec le concours de deux employés, à assurer le recouvrement d'effets de commerce et de créances litigieuses afférentes à des opérations antérieures. (Arrêt du Conseil d'Etat du 5 novembre 1934).
Ainsi que nous l'avons déjà signalé, les dégrèvements d'impôt foncier pour detles hypothécaires ou chirographaires ont été supprimés par les décrets-loi du 20 juillet 1934.

Toutefois, par mesure transiloire, l'Administration admet: que les intérêts payés en 1934 pourront encore faire l'objet d'une réclamation qui devra être présentée dans les trois premiers mois de 1935 .

$$
\text { *** }
$$

Aux termes de l'article 3 des textes codifiés par le décret du 20 juillet 1934, "les sociétés par actions et les sociétés à responsabilité limitée sont soumises à l'impôt sur les bénéfices industriels et commerciaux quel que soit leur objet ".

Ce texte, applicable à partir du $1^{\text {er }}$ janvier 1935 (bénéfices de 1934) a une portée considérable, car il atteint toutes les sociétés par actions ou à responsabilité limitée qui n'exercent aucune profession industrielle ni conmerciale: sociétés agricoles, sociétés immobilières se bornant à la gestion de leur patrimoine, sociétés d'enseignement, etc.

Toutefois, comme la situation à considérer est celle du $1^{\text {er }}$ janvier de l'année de l'imposition, les sociétés qui auraient changé de forme ou qui se seraient dissoutes antérieurement au $1^{\text {er }}$ janvier prochain ne tomberaient pas sous le coup de la nouvelle dispositions

L'Administration de l'Enregistrement a décidé que doivent être établis sur timbre les originaux des procès-verbaux d'assemblées générales d'actionnaires qui forment "le titre d'engagements réciproques, soit entre associés, soit entre les associés et la société \%. Tel est le cas, notamment, des procès-verbaux constatant la formation, la prorogation, la dissolution d'une société, une augmentation, une réduction de capital ou une fusion.

Par contre, l'exemption du droit de timbre de dimension est maintenue en ce qui concerne les procès-verbaux d'assemblées d'actionnaires qui relatent simplement des résolutions destinées à assurer la gestion sociale, par exemple la fixation et la répartition des bénéfices des exercices successifs.

Cette nouvelle règle n'est toutefois applicable qu'aux assemblées tenues posiérieurement au 14 avril 1934, date de la solution administrative qui l'a formulée. (Instruction 4171, \$34).

$$
* * *
$$

Aux termes du décret du 11 juillet 1934, la réduction à 12\% du tarif de l'impôt sur le revenu des capitaux mobiliers n'est accordée qu'aux produits distribués à des personnes physiques, à l'exclusion de ceux qui entrent dans le patrimoine de personnes morales non assujetties à l'impôt général sur le revenu.

Pour l'application de cette disposition, le Ministre des Finances a décidé, par solution du 20 août 1934, que les sociétés en nom collectif doivent être considérées, au point de vue físcal, comme 
des personnes physiques, et que les produits de capitaux mobiliers encaissés par elles doivent être imposés au tarif de $12 \%$, lorsque les conditions prévues par le décret du 11 juillet 1934 se trouvent par ailleurs toutes exactement remplies par ces prod'sits.$$
*^{*} *
$$

On sait que les rémunérations allouées aux associés-gérants des sociétés à responsabilité limitée et portées dans les frais et charges ne sont admises en déduction que si la majorité des parts sociales n'est pas possédée par l'ensemble des associés-gérants (Loi du 30 décembre 1928, article 4). Pour l'application de cette disposition, un associé-gérant co-propriétaire de parts sociales indivises doit être assimilé à un associé-gérant qui serait propriétaire unique d'un nombre de parts correspondant à ses droits dans l'indivision (Arrêt du Conseil d'Etat du 3 décembre 1934).

\section{***}

Le prix d'achat d'un droit au bail - achat réalisé en vue d'entreprendre un commerce différent de celui du précédent locataire - présente le caractère d'une dépense de premier établissement amortissable pour le calcul de l'impôt sur les qénéfices industriels et commerciaux (Arrêt du Conseil d'Etat du 19 novembre 1934 ).

$$
*^{*} *
$$

Lorsqu'une maison de commerce ayant son siège à l'étranger charge son représentant en France de répartir entre ses clients les marchandises qu'elle emmagasine dans un dépôt dont elle lui confie la gestion, cette maison est imposable en France à la patente et à l'impôt sur les bénéfices industriels et commerciaux, même si la livraison étant faite par le représentant, elle émet elle-même les factures et effectue drrectement les encaissements (Réponse du Ministre des Finances à la question écrite no 8404 posée par M. Fiori, député, $J$. O. du 4 novembre 1934, Chambre, débats, p. 2189).$$
* * *
$$

Le revenu des fonds confjés à un "trustee " par un père au profit de sa fille ne constitue pas une rente viagère imposable à la cédule des traitements et salaires (Arrêt du Conseil d'Etat du 3 décembre 1934).

Cet arrêt confirme la thèse - que nous avons soutenue dans une récente étude doctrinale - d'après laquelle les revenus perçus par le bénéficiaire ne perdent pas leur caractère propre et dojvent, par suite, être considérés comme des revenus d'immeubles, de valeurs mobilières françaises ou étrangères, de créances, etc., selon la nature des placements opérés par le " trustee ".

$$
\text { *** }
$$

Les contribuables relevant de la cédule des professions non commerciales doivent, en vertu de l'article 4 de la loi du 23 décembre 1933, tenir et communiquer à toute réquisition du contrôleur des contributions directes, un livre-journal présentant jour par jour le détail de leurs recettes professionnelles. Mais la communication de ces renseignements ne peut être exigée en ce qui concerne les opérations effectuées antérieurement à la date d'entrée en vigueur de la loi du 23 décembre 1933 (Réponse du Ministre des Finances à la question écrite no 8403 posée par M. Fiori, député, $J$. O. du 4 novembre 1934, Chambre, débats, p. 2189).
Dans une solution qu'elle vient de notifier par la voie de son Bulletin mensuel d'octobre, l'Administration de l'Enregistrement a décidé que les acomptes trimestriels dont le versement est obligatoire pour les sociétés doivent être calculés uniformément au tarif normal de $17 \%$ "lors même que la totalité des titres émis " par les Sociétés revêtiraient la forme nominative ".

Des termes dans lesquels est conçue ladite solution, il semble résulter que cette règle s'applique seulement aux valeurs négociables qu'elles soient d'ailleurs productives de revenus variables (actions, parts bénéficiaires) ou de revenus fixes (obligations).

Elle ne paraît pas devoir être étendue aux droits sociaux ou créances qui ne sont pas représentés par des titres négociables, c'est-à-dire notamment aux parts d'intérêt dans les sociétés en commandite simple, à responsabilité limitée ou civile, et les emprunts purs et simples. Pour ces droits sociaux ou créances dont la forme nominative est entièrement stable, l'Administration paraît admettre la possibilité de liquider les acomptes trimestriels au tarif de $12 \%$ à condition, bien entendu, que les ayants-droits soient des personnes physiques.

\section{$* * *$}

La question a été posée de savoir si, dans le cas d'une distribution gratuite d'actions et de négociation en bourse du droit à ces actions, il convient, pour déterminer le taux de l'impôt sur le revenu à appliquer, de considérer la personnalité du propriétaire du titre qui donne droit auxdites actions gratuites, auquel cas le taux serait de $12 \%$ s'il s'agit d'un propriétaire de titre nominatif remplissant les autres conditions prévues au décret, ou celle de l'acquéreur du droit négocié en bourse.

Il a été décidé que 1 z tarif de $12 \%$ est susceptible de bénéficier à la remise gratuite d'actions nouvelles, effectuée en vertu du droit de souscription appartenant encore ou ayant appartenu à celles des actions anciennes qui, au moment de l'incorporation des réserves au capital, avaient, depuis plus de six mois, la forme nominative (les autres conditions d'exemption étant supposées remplies par ailleurs).

Cette décision serait applicable alors même que le drojt de souscription aurait été détaché desdites actions nominatives anciennes et transmis par voie de négociation en bourse.

\section{$*^{*} * *$}

Lorsqu'un acte de partage qui prévoit le paiement d'une soulte à la charge de l'un de ses copartageants ne précise pas la date à laquelle cette soulte doit être payée, le jugement c'u tribunal qui, ul.érieurement a fixé cette date en s'inspirant à la fois des intentions des parties et des circonstances de fait, ne peut être interprêté comme emporiant prorogation d'un délai conventionnel antérieur.

Le droit proportionnel de prorogation n'est donc pas exigible (Cassation, 17 octobre 1934, aff. Montergon c- Enregistrement).

$$
*^{*} *
$$

Les états de traitements et de décompte dressés par un commerçant ou un industriel en vue du paiement des salaires de ses employés et ouvriers sont soumis au timbre des quittances lorsqu'jls sont émargés de la signature des ayants-droits.

Toutefois, si ces états sont signés plusieurs jours avant l'échéance, afin de constater simplement l'accord du créancier et du débiteur sur le montant des salaires dus et uniquement dans le but d'assurer la régularité des écritures, ils ne peuvent constituer le signe d'une libération qui, au moment de la signature, n'était que future et éventuelle; dans cette hypothèse, le droit de timbre-quittance n'est pas dû. 


\section{Taxe de transmission. - Valeur imposable Conséquences d'une augmentation de Capital}

Aux termes de la loi du 23 juin 1857, la taxe annuelle de transmission qui frappe les titres au porteur pour une année déterminée doit être calculée sur la valeur moyenne de ces titres durant l'année précédente, valeur fixée d'après la moyenne des cours pratiqués en Bourse, lorsqu'il s'agit de titres cotés, et par une déclaration estimative de la société dans l'hypothèse contraire.

Quant aux nouveaux titres émis par les sociétés, ils doivent être toujours assujettis à la taxe pendant la première année de leur existence, d'après leur valeur moyenne au cours de la même année, selon évaluation souscrite à la fin de chaque trimestre.

Parmi les difficultés auxquelles donnait lieu l'application de ce principe, l'une des plus délicates se présentait dans le cas où une société procède à une augmentation de son capital par l'émission, soit au pair, soit avec une prime, de nouvelles actions. La question se posait de savoir si cette opération a pour conséquence de modifier essentiellement les actions anciennes, à raison de la réduction de droits qu'elle leur a fait subir et si celles-ci peuvent être considérées comme des titres nouveaux.

Cette question avait été résolue par l'affirmative par le Tribunal de Béthune (13 mai 1925). Par contre, le Tribunal de Marseille (23 mai 1927, Cie Fraissinet et $C^{\text {le }}$ ) et celui de la Seine (1 ${ }^{\mathrm{er}}$ décembre 1928, Chargeurs Réunis) s'étaient prononcés pour la négative, conformément à la thèse de l'administration.

Mettant fin à cette controverse, la Chambre Civile de la Cour de Cassation vient de confirmer ces deux derniers jugements par deux arrêts en date du 20 novembre 1934, décidant que l'émission d'actions nouvelles, réalisée avec ou sans prime, n'emporte pas novation juridique des actions anciennes et que celles-ci doivent continuer à être imposées sur la base forfaitaire instituée par la loi, c'est-à-dire d'après leur cours moyen ou leur valeur moyenne durant l'année précédente.

Cette sentence est conforme à l'opinion que nous avons toujours soutenue.

\section{Retenues à pratiquer sur les salaires ou honoraires versés à des Contribuables domiciliés hors de France}

On a souvent déploré que notre système d'impôts sur les revenus qui atteint si durement les entreprises françaises, épargnât si souvent le produit de l'activité déployée en France par des étrangers n'ayant aucun domicile ni installation permanente sur notre territoire.

C'est pour mettre fin à cette inégalité que la loi du 6 juillet 1934 a posé le principe de la perception de l'impôt. cédulaire à la source sur les salaires et les honoraires encaissés en France par les personnes non domiciliées en France.

Cette " perception à la source " a été organisée par le décret-loi du 20 juillet 1934 et par le décret du 21 décembre 1934 ( $J . O$. du 25 décembre, page 12.704).

Bien qu'elles ne visent en fin de compte que les personnes ou sociétés installées à l'étranger, les dispositions nouvelles intéressent au plus haut point les entreprises françaises et plus généralement les personnes, sociétés ou associations payant des sommes imposables, puisque tous ceux qui versent lesdites sommes sont, depuis le $1^{\mathrm{er}}$ janvier 1935 , tenus sous leur responsabilité personnelle de retenir l'impôt correspondant et de le verser mensuellement au percepteur.

Désormais, les chefs d'entreprise devront donc avant de faire à une personne ou société installée à l'étranger un versement quelconque de salaires, pensions, honoraires, redevances, etc., rechercher s'il n'y a pas lieu à retenue de l'impôt correspondant.

\section{Versements susceptibles de donner lieu à retenue}

Sous réserve des exceptions dont il sera parlé ci-après, et qui découlent de conventions internationales, les versements susceptibles de donner lieu à retenue sont les suivants :
$1^{0}$ Traitements, indemnités, émoluments et salaires payés pour des services effectués en France à des personnes domiciliées hors de France (sauf les allocations aux familles nombreuses, sursalaire familial, allocations familiales, etc., versés uniquement par des employeurs ou groupements d'employeurs à leur personnel).

$2^{\circ}$ Pensions et rentes viagères servies à des personnes domiciliées hors de France (sauf les rentes viagères et allocations temporaires accordées aux victimes d'accidents du travail).

$3^{\circ}$ Honoraires payés en rémunération de l'activité déployée en France dans l'exercice d'une profession non commerciale (médecine, barreau, arts, sciences, etc.) à des personnes ou sociétés n'ayant pas d'installations professionnelles en France.

$4^{\circ}$ Bénéfices, revenus, produits et redevances réalisés en France par les mêmes personnes ou sociétés et rentrant dans la cédule des professions non commerciales, en vertu de l'article 78 du décret du 20 juillet 1934, c'est-à-dire notamment les revenus de la location du droit de chasse, les produits de la location du droit d'affichage, les redevances tréfoncières, les produits de droits d'auteurs et les produits perçus par les inventeurs au titre soit de la vente ou de la cession, soit de la concession de licences d'exploitation, de leurs brevets, marques de fabrique, procédés ou formules de fabrication.

\section{Calcul de l'impot à retenir}

La retenue doit, aux termes de la loi, être pratiquée sur "le montant net des sommes imposables". Ce montant net est déterminé en appliquant aux sommes payées une déduction forfaitaire de $20 \%$ s'il s'agit de traitements, salaires et autres 
rémunérations, ou d'honoraires, bénéfices, produits et redevances, et de $10 \%$ s'il s'agit de pensions et rentes viagères.

Pour le calcul de l'impôt, c'est-à-dire de la somme à retenir, il est appliqué au "montant net" calculé comme ci-dessus le taux de l'impôt correspondant à la nature du versement, soit $12 \%$ s'il s'agit d'un revenu non commercial, ou $6 \%$ s'il s'agit d'un traitement, d'une pension, d'une rente viagère.

On remarquera qu'en dehors de la déduction forfaitaire cidessus indiquée, il n'est fait aucune déduction à la base, ni aucune réduction pour charges de famille.

\section{Exceptions résultant de conventions internationales}

En raison des conventions qui lient la France à certains pays étrangers, l'obligation de la retenue n'existe pas en ce qui concerne les sommes énumérées ci-après :

$1^{\circ}$ Les sommes payées à titre de traitements, salaires, pensions ou rentes viagères ou de revenus non commerciauxx, à des contribuables ayant leur domicile en Sarre (convention francosarroise du 5 juillet 1922).

$2^{\circ}$ Les rentes viagères servies à des personnes ayant leur domicile fiscal en Belgique, les sommes payées à titre de revenus de professions non commerciales à des personnes ou sociétés ayant, dans le même pays, l'installation permanente où s'exerce l'activité d'où provient le revenu, ainsi que les autres revenus provenant de la cédule des professions non commerciales - en particulier les droits d'auteur et les revenus de brevets d'invention lorsque le bénéficiaire a son domicile fiscal en Belgique (Convention franco-belge du 16 mai 1931).

$3^{\circ}$ Les sommes payées dans les mêmes conditions à des personnes ou sociétés ayant leur domicile fiscal en Italie (Convention franco-italienne du 16 juin 1930).

$4^{0}$ Les sommes payées à titre de salaires à des travailleurs - et dans certains cas à titre d'honoraires à des médecins qui exercent leur activité en France et qui habitent solt en Luxembourg soit dans les zones frontalières belge et allemande, soit enfin dans les cantons suisses de Bâie-ville, Bâle campagne et Soleure.

\section{Sommes payées par des succursales situées hors de France}

Il n'y a pas lieu de pratiquer les retenues sur " les sommes dont le paiement entre dans les attributions d'établissements possédés hors de France par des entreprises ayant leur siège en France " et notamment sur "les arrérages de rentes viagères dépendant d'agences ou succursales exploitées hors de la métropole par les entreprises françaises d'assurances sur la vie".

\section{Formalités à remplir par les personnes ou sociétés effectuant des versements soumis à retenue}

$1^{0}$ Toute personne ou société effectuant des versements soumis à retenue doit de suite (car la loi est, rappelons-le, dès à présent applicable) demander par écrit, au contrôleur dont dépend le domicile de la personne ou le siège de l'établissement ou du bureau qui effectue les paiements soumis à retenue, la délivrance d'un carnet à souches numérotées et d'imprimés de "bordereaux-avis" destinés à accompagner ses futurs versements.

$2^{\circ}$ Elle est tenue, lors de chaque paiement donnant lieu à la perception à la source, d'opérer pour le compte du Trésor la retenue de l'impôt et de remettre à la partie prenante une quittance - exempte de timbre - de cet impôt, quittance détachée du carnet qui lui a été délivré, en ayant soin de porter sur la souche toutes les indications prévues par l'imprimé.

$3^{\circ}$ Dans les dix premiers jours de chaque mois, elle doit verser à la caisse du percepteur les retenues afférentes aux paiements qu'elle a opérés au cours du mois précédent; le versement est accompagné d'un "bordereau-avis " conforme aux indications de l'imprimé.

Les carnets doivent être tenus sans surcharges ni grattages; ils doivent être conservés pendant quatre ans et communiqués, à toute époque, aux agents des Contributions directes qui en font la demande.

Tout titulaire de carnet qui cesse d'en avoir l'emploi doit le présenter au contrôleur qui annule les reçus restant à utiliser ; tout titulaire qui transporte son domicile ou le siège de son établissement hors de la circonscription de contrôle dont il dépendait doit présenter le carnet au visa du contrôleur de la nouvelle circonscription.

\section{Responsabilité et sanctions}

Le code prévoit que les personnes ou sociétés assujetties à l'obligation de la retenue, qui n'auront pas effectué dans le délai prescrit les versements dont elles sont responsables ou qui n'auront fait que des versements insuffisants, seront personnellement redevables des droits non versés, majorés de $25 \%$. Ces droits seront perçus par voie de rôles qui pourront être mis en recouvrement jusqu'à l'expiration de la quatrième année suivant celle au cours de laquelle les revenus imposables auront été payés.

En raison de cette responsabilité, on ne saurait trop conseiller aux chefs d'entreprise qui seraient appelés à effectuer des versements soumis à retenue avant d'avoir reçu de leur contrôleur les imprimés nécessaires, de garder en compte la part revenant au Trésor, quitte à délivrer à la partie prenante uñe quittance extraite du registre à souche réglementaire dès réception de celui-ci.

Si l'on doit approuver pleinement le souci d'équité fiscale qui a inspiré les nouveaux textes, il est, par contre, permis de regretter que le contribuable et, plus particulièrement, le chef d'entreprise, se voie, en la circonstance, attribuer, contre son gré, le rôle de collecteur d'impôts et soit encore tenu à de nouvelles obligations d'une complexité toujours croissante.

\section{Extension des délais de contrôle de l'Administration et des sanctions fiscales en matières d'insuffisances de prix ou d'évaluations}

Nous avons donné précédemment l'analyse très sommaire des principales dispositions du décret du 20 juillet 1934, réalisant plusieurs modifications dans la législation fiscale de l'Enregistrement.

En raison de l'intérêt particulier qu'elle présente, nous croyons opportun de revenir rapidement sur l'une de ces réformes rela- tives aux insuffisances de prix ou d'évaluation d'immeubles ou de fonds de commerce, dans les actes de vente, les déclarations de succession, les actes de société, de partage, etc.

a) Extension du délai d'expertise :

Le droit d'expertise accorde à l'Administration de l'Enregistrement, en matière d'insuffisance de prix ou d'évaluation dans 
les actes ou déclarations constatant une mutation à titre onéreux ou à titre gratuit ou un échange d'immeubles, de funds de commerce, de navires cu de bateaux, devait, jusqu'à ce jour, s'exercer dans un délai maximum de deux ans, à compter du jour de l'enregistrement de l'acte de la déclaration. Ce délai était, en outre. réduit à six mois en matière de fonds de commerce (Loi du 13 juillet 1925, article 58)

L'article 8 du décret du 20 juillet 1934 élève respectivement ces délais à trois ans et un an. En d'autres termes, la requête en expertise de l'Administration pourra dorénavant être présentée dans les trois ans du jour de l'enregistrement de l'acte ou de la déclaration, s'il s'agit d'immeubles, de navires ou de baleaux, ou dans l'année de la même date, s'il s'agit de fonds de commerce.

Cette disposition est applicable dès à présent à toutes les mutations pour lesquelles le délai de prescription antérieure n'avait pris pas encore fin le 26 juillet $193^{\prime}$ '.

\section{b) Sanctions :}

Sous l'empire de la législation antérieure, l'insuffisance de prix ou d'évaluation, même égale ou supérieure au huitième du prix exprimé ou de la valeur déclarée, reconnue amiablement avant notification d'une requête en expertise, n'entraînait qu'un complément de droits simples sans pénalité.

Lorsque l'insuffisance reconnue amiablement au cours d'une expertise ou révélée par cette expertise était égale ou supérieure au huitième du prix exprimé ou de la valeur déclarée, elle entraînait : $1^{\circ}$ Le droit simple sur le complément d'évaluation ;

$2^{\circ}$ Un droit en sus, si l'insuffisance était reconnue amiablement avant le dépôt au greffe du Tribunal, du rapport de l'expert et un double droit en sus, dans le cas contraire;

$3^{\circ}$ Les frais de l'expertise.

Aucune pénalité n'était encourue et les frais de l'expertise restaient à la charge de l'Administration, lorsque l'insuffìsance, reconnue amiablement au cours de l'expertise ou révélée par cette expertise, était inférieure au huitième du prix exprimé ou de la valeur déclarée.

Tout en maintenant ces diverses dispositions, l'article $6 \mathrm{du}$ décret prérappelé frappe désormais d'un demi-droil cn sus les insuffisances autrefois exonérées de toute pénalité, reconnues amiablement avant la notification de la requête en expertise, lorsqu'elles sont égales ou supérieures au huitième du prix exprimé ou de la valeur déclarée.

Ces règles nouvelles, jointes au renforcement des cadres de contrôle, vont provoquer inévitablement, surtout en matière d'insuffisances de prix ou d'évaluation, un accroissement très sensible du nombre des contestations que soulève déjà trop souvent le Service de l'Enregistrement.

Il importera donc plus que jamais aux contribuables, notamment en cas d'acquisition, d'apport en société, de donation ou de succession, ayant pour objet un immeuble ou un londs de commerce, de faire procéder à une estimation préventive et de confier ce travail à un technicien qui, parfaitement au courant des méthodes administratives d'évaluation, soit à même, en cas de besoin, de défendre sa propre estimation avec les plus grandes chances de succès.

\section{Suppression du droit proportionnel de patente sur les locaux d'habitation}

Un décret du 30 novembre 1934 vient entin de réaliser, à partir du $1^{\text {er }}$ janvier 1935, la suppression du droit proportionnel de patente sur l'habitation, suppression dont le principe avait été posé, pour la seconde fois, par la loi du 6 juillet 1934 .

Cette réforme tant attendue intéresse la généralité des patentables.

Par contre, les mesures destinées, d'après la lò du 6 juillet 1934, à créer au profit des départements et des communes des ressources compensatrices n'ont encore été prises qu'en ce qui concerne les professions libérales.

\section{a) Dispositions intéressant la généralité des patentables.}

Dans la catégorie des locaux auxquels ne s'appliquera plus le droit proportionnel entrent, tout d'abord, ceux qui servent a l'habitation habituelle et principale des patentables exerçant seuls, des associés principaux, des sociétés en nom collectif et des préposés spéciaux visés à l'article 14 de la loi du 15 juillet 1880.

Le droit proportionnel cessera également d'être applicable aux divers locaux d'habitation (résidence secondaire des patentables, logement des associés secondaires, des sociétés en nom collectif, des directeurs de sociétés anonymes ou des gérants de sociétés à responsabilité limitée, des préposés, contremaîtres, surveillants, gardes-magasins, concierges, etc.) qui, d'après la loi ou la jurisprudence, étaient jusqu'alors passibles de ce droit lorsqu'ils pouvaient être considérés comme servant à l'exercice de la profession.
Il n'y aura, d'ailleurs, pas lieu de distinguer entre ceux de ces locaux qui étaient soumis au droit proportionnel d'après le taux spécial applicable à l'habitation et ceux qui étaient taxés d'après le taux prévu pour les locaux professionnels.

C'est ainsi, par exemple, que dans une entreprise, le logement occupé par un concierge chargé de surveiller l'entrée et la sortie des ouvriers devra, bien que, d'après la jurisprudence du Conseil d'Etat, il fût jusqu'alors imposable au droit proportionnel suivant le taux applicable aux locaux professionnels, être exonéré de ce dernier droit, au.même titre que la maison du directeur, qui devait être taxée d'après le taux prévu pour les locaux d'habitation.

Des indications qui précèdent, il résulte, en définitive, qu'un même local ne pourra plus, en principe, donner lieu à l'application simultanée de la contribution mobilière et du droil proportionnel de patente, même si ces deux impôts sont établis au nom de contribuables distincts. Exception est faite, bien entendu, des locaux affectés à la location en meuble, dont la valeur locative sert do basse à la patente due par le loueur et dont les occupants sont, le cas échéant, passibles de la contribution mobilière.

La suppression du droit proportionnel d'habitation met fin à une double injustice. Elle libère tout d'abord les commerçants isolés et les associés en nom d'une lourde charge que n'avaient jamais supporté les administrateurs-délégués des sociétés anonymes ni les gérants des sociétés à responsabilité limitée.

Elle évite, d'autre part, de faire payer plus ongtemps aux 
pères de famille nombreuse - obligés, du fait même de leur situation de famille, de se loger plus grandement - des impôts beaucoup plus lourds que ceux qu'avaient supporté les célibataires.

\section{b) Dispositions spéciales aux professions libérales.}

En ce qui concerne les professions libérales le nouveau décret compense, dans une certaine mesure, la suppression du droit proportionnel de patente sur l'habitation par la création d'un "droit fixe ", analogue à celui que payent déjà les commerçants.

Ce droit fixe sera, en principal, de 140 francs à Paris, de 100 francs dans les communes dont la population dépasse 100. n00 âmes, de 80 francs dan, les communes de 50.000 à 100.000 âmes, et il variera de 60 à 18 francs dans les localités moins importantes.

En outre, le droit proportionnel sera fixé au dixième de la valeur locative des locaux professionnels, alors que, jusqu'ici. il n'atteignait, selon les cas, que le douzième ou le quinzième de la valeur locative totale des locaux imposables.

On remarquera d'ailleurs que le droit fixe ainsi créé et la majoration du droit proportionnel ne frapperont pas seulement les professions énumérées au décret (architectes, avocats, ingénieurs civils, médecins, notaires, etc.), mais également toutes celles qui ont fait l'objet d'une " assimilation " avec ces dernières. C'est ainsi que les "conseils juridiques", bien que ne figurant pas sur la liste insérée dans le nouveau texte se trouveront traités comme les "avocats" auxquels ils sont déjà assimilés pour l'application des droits de patente.

Malgré ces dispositions aggravantes, la réforme se traduira encore, dans la plupart des cas, par une économie sensible pour les patentables exerçant une profession libérale.

Un exemple chiffré le fera mieux comprendre.
Considérons, par exemple, le cas d'un contribuable qui, exerçant à Paris une profession libérale, celle de médecin par exemple, dispose de locaux professionnels d'une valeur locative de 10.000 francs et occupe, pour son habitation personnelle, un appartement d'un loyer de 14.000 francs.

Les droits de patente qui lui étaient réclamés antérieurement à la réforme atteignaient le douzième de la valeur locative tant de son cabinet médical que de son appartement, soit: 24.000

$\frac{12}{12}=2.000$ francs en principal, chiffre qui, multiplié par

le centime-le-franc de 8 environ, donnait une patente effective de 16.000 francs.

Sous le nouveau régime, la patente s'établira comme suit :

Droit fixe. 140 francs

Droit proportionnel au $10^{\mathrm{e}}$ sur les locaux professionnels seulement $(10.000: 10) \ldots \ldots . .1 .000 "$

Total des droits en principal 1.140 francs

Montant effectif de la patente:

$$
1.140 \times 8 \text { environ }=9.120 \text { francs. }
$$

La suppression du droit proportionnel d'habitation se traduit donc, pour le patentable pris comme exemple, par une économie annuelle de $(16.000-9.120) 6.880$ francs.

$$
*^{*} *
$$

Saluons donc avec satisfaction la suppression du droit proportionnel sur l'habitation qui apporte enfin un peu plus d'équité dans notre vieille égislation des patentes.

\section{INFORMATIONS}

\section{Le prochain Congrès de la Conférence Internationale des Grands Réseaux Electriques}

Ce Congrès, qui est l'une des manifestations périodiques les plus importantes de l'électrotechnique mondiale, et auquel sont présentées tous les deux ans les nouveautés les plus sensationnelles de la technique, aura lieu à Paris du jeudi 27 juin au samedi 6 juillet.

La crise semble, jusqu'ici, n'avoir aucune influence sur son succès habituel, puisqu'au 15 mars dernier il y avait déjà, comme pour les sessions précédentes, plus de 350 inscriptions recueillies, provenant de 21 pays différents, ce qui permet de prévoir que l'effectif du Congrès sera vraisemblablement de 700 participants au moins (non compris les personnes qui accompagnent les congressistes).

Il y aura 150 rapports traitant les sujets les plus actuels de la technique et qui apporteront, comme d'habitude, une véritable mine de précieux et vivants renseignements.
Les langues utilisées au Congrès sont les principales langues usuelles de la technique, notamment l'allemand, l'anglais, le français et l'italien ; l'organisation du Congrès est telle que toute personne connaissant l'une de ces langues peut suivre avec commodité et fruit la totalité des discussions.

Le droit d'inscription est de 375 francs français, moyennant quoi les congressistes recevront la collection complète des rapports, pourront assister à la totalité des séances et participer aux visites, réceptions et voyages organisés en leur faveur.

Les Chemins de fer français accordent une réduction de $40 \%$ en faveur des congressistes et des personnes qui les accompagneront.

La Conférence a obtenu pour les congressistes des prix forfaitaires extrêmement réduits dans les meilleurs hôtels : 175 francs. pour 5 jours, 300 francs pour 10 jours.

Pour tous renseignements, prière de s'adresser au Secrétariat du Congrès, 54, avenue Marceau, Paris. 
Enquête sur les travaux publics nationaux. L'Organisation des Communications et du Transit de la Société des Nations vient de publier un volume de près de 300 pages sous le titre : Enquête sur les travaux publics nationaux. - Prix: 12 fr. suisses.

On se rappellera que c'est à la suite d'une initiative de I'Organisation internationale du Travail signalant l'intérêt que pourrait offrir, par rapport à la crise économique et au chômage, l'élaboration de programmes internationaux de travaux publics, que des études ont été entreprises sur les questions de travaux publics.

Le Conseil de la Société des Nations, en prenant connaissance, en septembre 1933, de l'activité de l'Organisation des Communications et du Transit dans ce domaine, constata l'utilité d'un examen international continu des questions générales de travaux publics, y compris les grands programmes de travaux exécutés par des moyens nationaux, en vue de confronter les expériences acquises dans les divers pays en ce qui concerne les effets de l'exécution de travaux publics sur la reprise de l'activité économique et sur le chômage. Aussi, le Conseil fut-il d'avis de procéder à la réunion d'une documentation préparatoire qui pourrait être nécessaire pour des discussions ultérieures.

Un rapport adopté par l'Assemblée en 1933 exprima des vues analogues. La documentation envisagée devrait permettre aux Gouvernements de juger de la possibilité et de l'opportunité de poursuivre, dans les circonstances actuelles, une politique d'exécution parallèle de leurs programmes de travaux publics. L'Assemblée estima que dans la période de détresse que connait le monde, cette question ne pourrait manquer d'intéresser grandement l'opinion publique et les gouvernements de la plupart des pays.

Conformément aux vues de l'Assemblée et du Conseil, le Secrétaire général demanda alors aux Gouvernements de lui fournir les renseignements voulus, en se basant sur un questionnaire conçu de manière à laisser une certaine latitude pour les réponses.

Les réponses de vingt-neuf gouvernements des pays dont les noms suivent sont reproduites, généralement "in extenso", dans le volume qui vient de sortir de presse.

Union Sud-Africaine, Australie, Autriche, Belgique, RoyaumeUni de Grande-Bretagne et d'Irlande du Nord, Bulgarie, Canada, Danemark, Estonie, Etats-Unis d'Amérique, Finlande, France, Grèce, Haïti, Irak, Italie, Lettonie, Lithuanie, Luxembourg, Nicaragua, Norvège, Nouvelle-Zélande, Pays-Bas, Portugal, Salvador, Suisse, Tchécoslovaquie, Turquie, Yougoslavie.

L'enquête a porté sur: les travaux publics entrepris dans les divers pays depuis le début de 1929 (achevés, en cours d'exécution ou prévus); sur les principales méthodes administratives adoptées pour l'exécution de ces travaux; sur les principales méthodes de financement; sur la répartition des dépenses destinées à l'exécution de ces travaux entre les matériaux et l'outillage d'une part et la main d'œuvre d'autre part; sur l'appréciation par le Gouvernement intéressé des effets obtenus ou escomptés quant à la reprise de l'activité économique ou industrielle et quant au chômage. Les Gouvernements ont été invités à opérer la classification des travaux selon les catégories suivantes : routes et ponts ; voies ferrées; bonification agricole; canaux et autres voies d'eaux intérieures ; amélioration foncière ; adduction d'eau potable et évacuation des eaux d'égouts ; travaux dans les ports maritimes et fluviaux; établissement d'aéroports ; travaux de bâtiments ; installations électriques; usines à gaz et distribution de gaz; installations télégraphiques; téléphoniques et stations de radiodiffusion ; divers.

Cette étude sur les travaux publics nationaux est la première qui ait été basée sur des renseignements officiels demandés à l'ensemble des Gouvernements. La documentation abondante contenue dans le volume est de nature à intéresser les autorités compétentes et l'opinion publique des divers pays.

Applications Agricoles et rurales de l'électricité, par M. Vignerot, Ingénieur en chef du Génie rural; L. PATrix, Ingénieur principal du Génie rural, Professeur à l'Ecole Supérieure du Génie rural; G. DABAT, Ingénieur principal du Génie rural. - Un volume $(16,5 \times 25)$ de 334 pages, 280 figures. Prix : 40 fr. Librairie de l'Enseignement Technique, L. Eyrolles, éditeur. En vente à la librairie Arthaud, 23, Grande-Rue, Grenoble.
L'électrification des campagnes françaises, entreprise sur une vaste échelle en 1920, au lendemain de la grande guerre, peut maintenant être considérée comme achevée dans la plupart des départements ; on évalue à plus de 6 milliards de francs le montant des travaux d'électrification rurale exécutés au 31 décembre 1934 et à 27.500 le nombre des communes, rurales qui, depuis 1920 , ont été dotées d'une distribution d'énergie électrique.

Mais l'électrification des campagnes n'aura pleinement atteint son but que le jour où le cultivateur emploiera largement dans sa ferme l'électricité mise à sa disposition.

Certes, dans beaucoup de régions, le courant est malheurcusement encore trop cher et les agriculteurs se bornent, le plus souvent, à utiliser l'électricité pour l'éclairage.

G'est par l'éducation des populations et par un aménagement judicieux des tarifs que l'on provoquera la généralisation souhai table des emplois de l'électricité à la campagne.

Pour aider à atteindre ce but, les auteurs se sont attachés à mettre en évidence les commodités et les avantages que les usagers peuvent attendre des applications bien comprises de l'électricité (éclairage rationnel des bâtiments de la ferme, applications domes tiques, applications agricoles et rurales). Ils ont notamment longuement étudié les conditions d'adaptation du moteur électrique à la commande des diverses machines agricoles. Enfin, dans un chapitre spécial qui traite des questions relatives à la vente de l'énergie électrique, ils préconisent de nouveaux modes de tarification bien adaptés aux besoins des agriculteurs.

Leur exposé, mis à la portée de tous, ne suppose aucune connais sance technique spéciale; il s'adresse à la fois aux cultivateurs et aux petits artisans des campagnes, aux ingénieurs, aux distributeurs et aux chefs de secteurs ruraux, aux installateurs électriciens, ainsi qu'à toutes les personnes qui, à des titres divers, s'intéressent aux questions d'économie rurale.

\section{Une intéressante publication sur " Le rail d'autrefois ».}

Soas ce titre, vient de paraître un numéro spécial du Bulletin P.-L.-M., la revue illustrée que ce Réseau publie depuis 1929 .

Comme le dit, en préface, M. Mugniot, Directeur général de la Compagnie, ce numéro spécial constitue la réédition et la mise à jour d'un fort bel ouvrage "Hommes et choses du P.L.-M. " qu'avait publié la Compagnie en 1910 et qui "a été si apprécié qu'il est, depuis longtemps, introuvable".

Faut-il dire que cette nouvelle publication, qui s'agrémente, comme l'édition ancienne, de fort belles gravures d'autrefois, ne présente pas seulement un intérêt d'ordre rétrospectif " Au lecteur attentif elle apparaîtra, au contraire, d'une grande actualité, en ce sens qu'en étudiant l'histoire du P.-L.-M., en passant en revue toutes les difficultés qu'il a réussi à surmonter dans le passé, on est conduit à penser qu'il saura de même triompher des difficultés présentes.

Voici quels sont les titres des chapitres retraçant l'attachante " carrière " de cette grande entreprise ferroviaire:

L'Ingénieur Beaunier (créateur de Saint-Etienne-Andrézieux, Ie plus ancien chemin de fer de France). Les Frères Seguin. Paulin Talabot. La crise de 1845, la Révolution de 1818 et le Second Empire, 1870-1871. Le Plan Freycinet et les Conventions de 1883. Le cinquantenaire de la Compagnie (1907) et la période précédant la guerre. De la guerre de $1914 \grave{a}$ nos jours, dernier chapitre particulièrement intéressant où sont évoqués les travaux de rééquipement technique de l'après-guerre ainsi que les efforts actuels de coordination des transports et d'action commerciale.

Cette édition ne fera pas qu'intéresser, elle amusera aussi, car les chápitres qui viennent d'être cités - surtout ceux du début, visant la période héroïque des chemins de fer - foisonnent de détails pittoresques.

Signalons en terminant que le Bullelin P.-L.-M. est en vente, au prix de 2 francs, dans les bibliothèques des principales gares de ce Réseau ainsi qu'à la Maison de France (101, Champs-Elysées) et à l'Agence P.-L.-M. de Renseignements (88, rue Saint-Lazare). Il peut être également adressé par la Rédaction du Bulletin P.-L.-M., 88, rue Saint-Lazare, Paris, contre envoi de la somme de 2 francs. 
Pour la thermodynamique. Aide-calcul graphique, par Coloman VARGHA, Ingénicur, avec préface de M. Ch. SEgurn, Inspecteur général de l'Aéronautique. - Un atlas $(27 \times 37)$ de 28 planches, en un carton. Prix : 115 fr. - Librairie Polytechnique Ch. Béranger, éditeurs. En vente à la librairieArthaud, 23, GrandeRue, Grenoble.

M. Coloman Vargha a publié en 1930, à la Librairie Polytechnique Ch. Béranger, sous le titre d'Aide-calcul graphique pour la mécanique générale, une série d'abaques pour le calcul des éléments des machines.

Le nouveau travail nomographique qu'il présente aujourd'hui concerne les calculs relatifs au fonctionnement des moteurs et compresscurs.

Le Service des Recherches de l'Aéronautique ne pouvait qu'accueillir avec faveur cet ouvrage qui se rattache, par son objet, à ceux qui sont particulièrement susceptibles de l'in.éresser.

Il contient une série d'abaques pour le calcul du volume, de la pression et de la température d'une masse de gaz soumise à des actions mécaniques et thermiques. D'autres permettent de déterminer l'énergie mise en jeu dans les transformations d'un fluide, ou les conditions de son écoulement. On y remarquera l'artifice qui permet de traiter par un seul système de tracés les problèmes de compression et de détente pour toutes les valeurs usuelles du coefficient polytropique en adoptant une terminologie technique pratique) et une série d'abaques pour le calcul du temps de vidange et de remplissage des réservoirs à gaz, problème compliqué pour la solution duquel on trouve peu de documents pratiques. On y trouvera enfin des abaques rattachant la composition des gaz de l'émission des moteurs thermiques à la composition du carburant et à la proportion d'air comburant: ils peuvent être précieux pour l'analyse rapide des essais.

Les abaques de cette publication touchent donc à bien des questions dont la solution importe au constructeur comme à l'utiliseur des machines. Leur caractère, comme l'avait déjà montré M. J.-L. Breton, membre de l'Institut, dans la préface qu'il a écrite dans le premier ouvrage de M. Vargha, est nettement pratique. Il existe dans la littérature technique française des ouvrages magistraux sur la théorie des abaques : ils ont pour but d'apprendre au technicien comment il doit s'y prendre pour construire un abaque représentant les résultats numériques de l'application d'une formule ou de données résultant de l'expérience, et, dans ces conditions, ils montrent toutes les ressources que fournit la géométrie pour adapter les tracés des types les plus divers aux cas les plus variés. Le but de $M$. Vargha est plus directement utilitaire: se cantonnant dans un type particulier de tracés, celui des abaques cartésiens accolés à échelles logarithmiques, il cherche à enfermer dans ce type de tracés et sous une forme très homogène, le traitement d'un grand nombre de problèmes d'intérêt pratique.

Une telle méthode est essentiellement propre à la vulgarisation du calcul graphique, car un technicien qui s'est servi une première fois des abaques de $M$. Vargha pour résoudre un problème particulier; n'éprouve plus ni embarras ni hésitation à employer ces procédés dans tout autre domaine.

L'auteur a eu, avant tout, le souci de rendre vraiment pratique et facile l'emploi des abaques qu'il a établis. Il a parfaitement réussi dans cet effort et doit en être loué sans réserve.

SEGUIN,

Directeur du Service des Recherches de l'Aéronautique.

Théorie du circuit électrique de Heaviside (Applications aux filtres électriques, aux cables sous-marins, aux lignes de transmission d'énergie et aux lignes artificielles), par M. Louis CoHen, Ingénieur conseil, Professeur d'Electrotechnique à l'Université George Washington. Traduit de l'anglais par Frédéric Sarrat, Directeur général honoraire et Administrateur de la Société "Electricité et Gaz du Nord ". - Un volume $(16,5 \times 25)$ de 192 pages. Prix : 30 fr. - Librairje de l'Enseignement Technique L. Eyrolle, éditeur. En vente à la librairie Arthaud, 23, Grande-Rue, Grenoble.

M. Ie Professeur Louis Cohen, bien connu pour ses remarquables contributions au Bulletin du "Bureau of Standards " de Washington, a eu le rare mérite d'exposer dans ce livre, sans faire appel à des connaissances mathématiques trop élevées, la théorie du circuit électrique de Heaviside, basée sur le "calcul opératoire" et le célèbre "théorème de développement ".
M. Cohen a mis ainsi à la portée des ingénieurs, dans un traité didactique d'une lecture aisée, les méthodes de calcul si originales et si fécondes de ce grand savant.

Les multiples problèmes traités dans cet ouvrage, par cette théorie, notamment en ce qui concerne les circuits-filtres, les câbles sous-marins, les lignes de transmission d'énergie et les lignes artificielles montrent toute la généralité de l'application de cette théorie aux cas les plus variés.

Les méthodes de Heaviside sont particulièrement précieuses par leur puissance et leur rapidité dans l'étude des questions difficiles, telles que la propagation des phénomènes périodiques ou des perturbations dans les câbles ou les lignes de transmission.

Ce livre, dont aucun ouvrage correspondant n'existe actuellement dans la littérature technique française, paraît donc destiné à rendre les plus grands services aux nombreux ingénieurs s'occupant de transmissions d'énergie, de communications téléphoniques ou télégraphiques, ou de services de radio-diffusion.

Les réseaux de transmission d'énergie (Réglage et stabilité. Surintensités. Surtensions. Protection sélective), par Jean Falrou Docteur ès-Sciences, Ingénieur E. S. E., Ingénieur-conseil à l'Union d'Electricité. - Un volume in-8 $(25 \times 16)$ de 558 pages, avec 288 figures. Prix : 125 fr. - Librairie Gauthier-Villars, éditeurs. En vente à la librairie Arthaud, 23, Grande-Rue Grenoble.

Cet ouvrage traite des principaux problèmes que soulèven l'établissement et l'exploitation des réseaux de transmission d'énergie. Il est divisé en quatre parties distinctes dont aucune ne saurait prétendre épuiser le sujet auquel elle est consacrée, mais dans lesquelles l'auteur a rassemblé les principes fondamentaux nécessaires à leur compréhension en s'inspirant des théories les plus modernes.

Dans la première partie, réservée à l'étude générale des systèmes de transmission d'énergie, se trouve exposée une théorie complète du fonctionnement des lignes. Cette théorie sert d'introduction à une discussion méthodique des différents systèmes de transmission ainsi qu'à l'étude de la régulation des réseaux développée par la suite. La stabilité des réseaux en régime permanent et en régime troublé est étudiée en détail dans cette première partie.

La seconde partie est consacrée à l'étude des surintensités. Après avoir développé une méthode de calcul des courants de court-circuit, l'auteur a consacré un chapitre spécial aux différents problèmes relatifs aux effets nuisibles des surintensités : échauffements anormaux, efforts électrodynamiques, et surtensions induites dans les lignes de télécommunication. L'étude des moyens propres à limiter l'amplitude des courants de court-circuit, et l'examen des différentes solutions adoptées pour la mise à la terre du point neutre, font ensuite l'objet d'assez longs développements. La seconde partie se termine par une description de quelques appareils de mesure et de contrôle permettant d'enregistrer les courts-circuits.

La troisième partie se rapporte à l'étude des surtensions, et, d'une manière plus générale, à celle des régimes transitoires ou permanents auxquels elles sont dues. On y trouvera d'abord l'établissement d'un certain nombre d'expressions analytiques qui interviennent continuellement dans les calculs des phénomènes transitoires, puis l'indication des grandeurs caractéristiques qu'il faut attribuer aux éléments constitutifs d'un réseau pour qu'on puisse les assimiler à des circuits simples. L'auteur analyse ensuite un certain nombre de surtensions, telles que celles que peuvent occasionner les manœuvres d'interrupteur, les résonances, les arcs à la terre, etc. Un chapitre spécial est consacré à l'étude de l'enregistrement des surtensions au moyen du klydonographe.

La quatrième partie traite des systèmes de protection sélective à un point de vue général, c'est-à-dire en considérant les principes plutôt que les détails de réalisation des appareils utilisés. On y trouvera une description de nombreux dispositifs quì ont déjà fait l'objet d'applications et reçu la sanction de l'expérience, ainsi qu'une discussion de leurs avantages et inconvénients respectifs.

Le Gérant: A. CRÉré.

Anciens Etablissements Legendre, 12-14, rue Bellecordière Lyon 